\title{
Avaliação do potencial da madeira de Pinus patula Schltdl. \& Cham para a produção de painéis de madeira aglomerada
}

\author{
Pinus patula Schltdl. \& Cham wood potential \\ evaluation for particleboard production
}

\author{
Polliana D'Angelo Rios ${ }^{1}$, Giuliano Ferreira Pereira ${ }^{2}$, Helena Cristina Vieira ${ }^{3}$, \\ Willian Grubert ${ }^{3}$, Alexsandro Bayestorff da Cunha ${ }^{1}$ e Martha Andreia Brand ${ }^{1}$
}

\begin{abstract}
Resumo
Neste trabalho objetivou-se avaliar o potencial de utilização da madeira de Pinus patula como alternativa para a produção de painéis de madeira aglomerada, além de analisar o efeito da utilização de diferentes teores do adesivo ureia formaldeído sobre as propriedades dos painéis. Foram utilizados oito tratamentos, sendo quatro compostos por Pinus patula e quatro de Pinus spp. utilizando ureia formaldeído como adesivo, nas proporções $6,8,10$ e $12 \%$ para cada espécie. Os painéis foram produzidos com densidade nominal de $650 \mathrm{~kg} \cdot \mathrm{m}^{-3}$, as dimensões de $40 \times 40 \times 1,6 \mathrm{~cm}$ (largura, comprimento e espessura), ciclo de prensagem com pressão de $40 \mathrm{kgf} / \mathrm{cm}^{2}$, temperatura de $180^{\circ} \mathrm{C}$ e 8 minutos de duração. Na análise química da madeira foram determinados os teores de extrativos totais, cinzas, lignina e holocelulose. A densidade média obtida para os painéis produzidos foi de $565 \mathrm{~kg} \cdot \mathrm{m}^{-3}$. As propriedades físicas analisadas nos painéis aglomerados foram: densidade do painel, absorção de água (AA) após 2 e 24 horas e inchamento em espessura (IE) após 2 e 24 horas. Os testes mecânicos realizados foram: módulo de elasticidade à flexão estática (MOE), módulo de ruptura à flexão estática (MOR) e ligação interna (LI). Ao avaliar as características químicas da madeira de Pinus patula e os resultados dos testes físicos e mecânicos dos painéis produzidos, pode-se observar que os resultados são estatisticamente iguais ou superiores aos obtidos para Pinus spp. (Industrial) e semelhantes aos encontrados na literatura, com exceção das propriedades de Módulo de elasticidade e Módulo de ruptura. Além disso, destaca-se que os painéis de Pinus patula confeccionados com $12 \%$ de ureia formaldeído apresentaram o maior potencial de utilização.
\end{abstract}

Palavras-chave: Painéis de Madeira, Resina sintética, Espécie não-convencional.

\begin{abstract}
This work aimed to evaluate the potential use of Pinus patula as an alternative for chipboard panel production, and to analyze the effect of using different amounts of urea formaldehyde adhesive on the panels' properties. Eight treatments were used, four composed of Pinus patula and four of Pinus spp. using urea formaldehyde as the adhesive in the proportions of $6,8,10$ and $12 \%$ for each species. The panels were produced with nominal density of $650 \mathrm{~kg}^{-3}$, and dimensions of $40 \times 40 \times 1,6 \mathrm{~cm}$ (width, length and thickness); the pressing cycle with pressure of $40 \mathrm{kgf} / \mathrm{cm}^{2}$ of pressure; $180^{\circ} \mathrm{C}$ of temperature 8 minutes long. In the wood chemical analysis the total content of extract, ash, lignin and holocelulose was determined. The average density obtained for the panels produced was $565 \mathrm{~kg} \cdot \mathrm{m}^{-3}$. The physical properties examined of the particle boards were: Panel density, water absorption (AA) after 2 and 24 hours, and thickness swelling (IE) after 2 and 24 hours. The mechanical tests performed were: modulus of bending (MOE), modulus of rupture in bending (MOR) and internal bond (LI). To evaluate the chemical characteristics of Pinus patula and the results of physical and mechanical tests of panels produced, it can be seen that the results are statistically equal or superior to those obtained for Pinus spp. (industrial) and similar to those found in the literature, except for the modulus properties and rupture module. In addition, it is emphasized that the panels of Pinus patula made with $12 \%$ urea formaldehyde showed the greatest potential for use.
\end{abstract}

Keywords: Wood paneling, synthetic resin, unconventional species.

\footnotetext{
1Professor(a) Adjunto(a) do Departamento de Engenharia Florestal. UDESC - Universidade do Estado de Santa Catarina. Av. Luiz de Camões, 2090 - Conta Dinheiro - 88520-000 - Lages, SC - Brasil. E-mail: polliana.rios@udesc.br; a2abc@cav.udesc.br; a2mab@cav.udesc.br.

${ }^{2}$ Mestre em Engenharia Florestal. UDESC - Universidade do Estado de Santa Catarina. Av. Luiz de Camões, 2090 - Conta Dinheiro - 88520-000 - Lages, SC, Brasil. E-mail: giuliano.giiu@gmail.com.

${ }^{3}$ Mestrando(a) em Engenharia Florestal. UDESC - Universidade do Estado de Santa Catarina. Av. Luiz de Camões, 2090 Conta Dinheiro - 88520-000 - Lages, SC - Brasil. E-mail: lenacristin@hotmail.com; w.grubert@live.com.
}

Sci. For., Piracicaba, v. 44, n. 110, p. 497-508, jun. 2016 DOI: dx.doi.org/10.18671/scifor.v44n110.22 
Rios et al. - Avaliação do potencial da madeira de Pinus patula

Schltdl. \& Cham para a produção de painéis de madeira aglomerada

\section{INTRODUÇÃO}

A espécie é uma das variáveis de maior importância no processo de confecção de painéis de madeira aglomerada de média densidade (MDP) uma vez que interfere significativamente na qualidade do mesmo. Em geral madeiras de baixa densidade são mais adequadas, pois aumentam a razão de compactação e desse modo concedem melhores características mecânicas aos painéis produzidos (IWAKIRI et al, 2002).

Por apresentarem baixa densidade e crescimento rápido, as espécies mais utilizadas no Brasil para a confecção de painéis de madeira aglomerada são as do gênero Pinus (ABRAF, 2010). Contudo, estudos relacionados à utilização do Pinus patula para a produção de painéis de madeira aglomerada ainda são raros.

A espécie Pinus patula tem apresentado crescimento satisfatório em povoamentos industriais ao longo dos trópicos e sub-trópicos, com destaque para a África do Sul onde figura como a espécie de conífera mais cultivada (DAFF, 2010).

No Brasil, o melhor desenvolvimento do Pinus patula ocorre em regiões de altitude elevada como no Oeste catarinense, Sudoeste do Paraná e Nordeste do Rio Grande do Sul. Nessas regiões a espécie é encontrada em plantios experimentais e pode apresentar taxas de incremento maior que a do Pinus taeda (SHIMIZU, 2005).

Outra variável de grande importância para a produção de painéis de madeira aglomerada é o adesivo, chegando a representar 50\% do custo total de produção. Segundo Carvalho et al. (2014), a maior parte os painéis de madeira aglomerada produzidos no Brasil são confeccionados com a ureia formaldeído. Por ser o componente de maior custo, a definição da quantidade de adesivo a ser utilizada torna-se bastante relevante, no sentido de se buscar uma otimização na relação custo-benefício (MENDES et al., 2012).

Em decorrência desses fatores, fica evidente a relevância de estudos que avaliem diferentes parâmetros envolvidos na produção de painéis de madeira aglomerada, como matérias-primas e sua correlação com as propriedades dos painéis. Concomitantemente, esses estudos podem incentivar a utilização de novas espécies e processos no meio industrial.

Nesse contexto, o presente estudo tem como objetivo avaliar o potencial da madeira de Pinus patula para a produção de painéis de madeira aglomerada e analisar o efeito da utilização de diferentes teores do adesivo ureia formaldeído sobre as propriedades dos painéis.

\section{MATERIAL E MÉTODOS}

\section{Coleta e preparo do material}

As árvores de Pinus patula, com diâmetro à altura do peito (DAP) médio de 21,42 cm e altura média de $16,70 \mathrm{~m}$, foram retiradas de um povoamento localizado no Município de Campo Belo do Sul, no Estado de Santa Catarina (Latitude $27^{\circ} 53^{\prime}$ 55" Sul e Longitude 50 45' 26" Oeste). Os indivíduos foram seccionados em toras de 1,5 metros até a altura comercial. As toras foram transformadas em cavacos por meio de um picador industrial. Para transformar os cavacos em partículas, utilizou-se um moinho de facas do tipo Willey com peneiras de 3,00 $\mathrm{mm}$. Por sua vez, a madeira do Pinus spp. foi fornecida por uma empresa da região do Planalto Catarinense, na forma de partículas. Na Figura 1 estão apresentadas as partículas de Pinus patula e Pinus spp.

Posteriormente, com as partículas obtidas, foram produzidos painéis aglomerados e realizados os testes físicos e mecânicos.

\section{Análise química}

A análise química do material foi realizada para melhor discutir os resultados encontrados nesse trabalho. Na padronização das amostras para a análise, foram utilizadas as peneiras de 40/60 Mesh, sendo selecionadas as partículas que passaram pela peneira de 40 Mesh e ficaram retidas na de 60 Mesh. Após a padronização dimensional, as amostras foram mantidas em sala de climatização, com umidade relativa de $60 \pm 5 \%$ e temperatura de $20 \pm 3^{\circ} \mathrm{C}$. Determinou-se a porcentagem de extrativos totais, o teor de cinzas e lignina, utilizando as normas NBR14660 (ABNT, 2004), TAPPI T222 (TAPPI, 2002) e TAPPI T413 (TAPPI, 1993), respectivamente. 


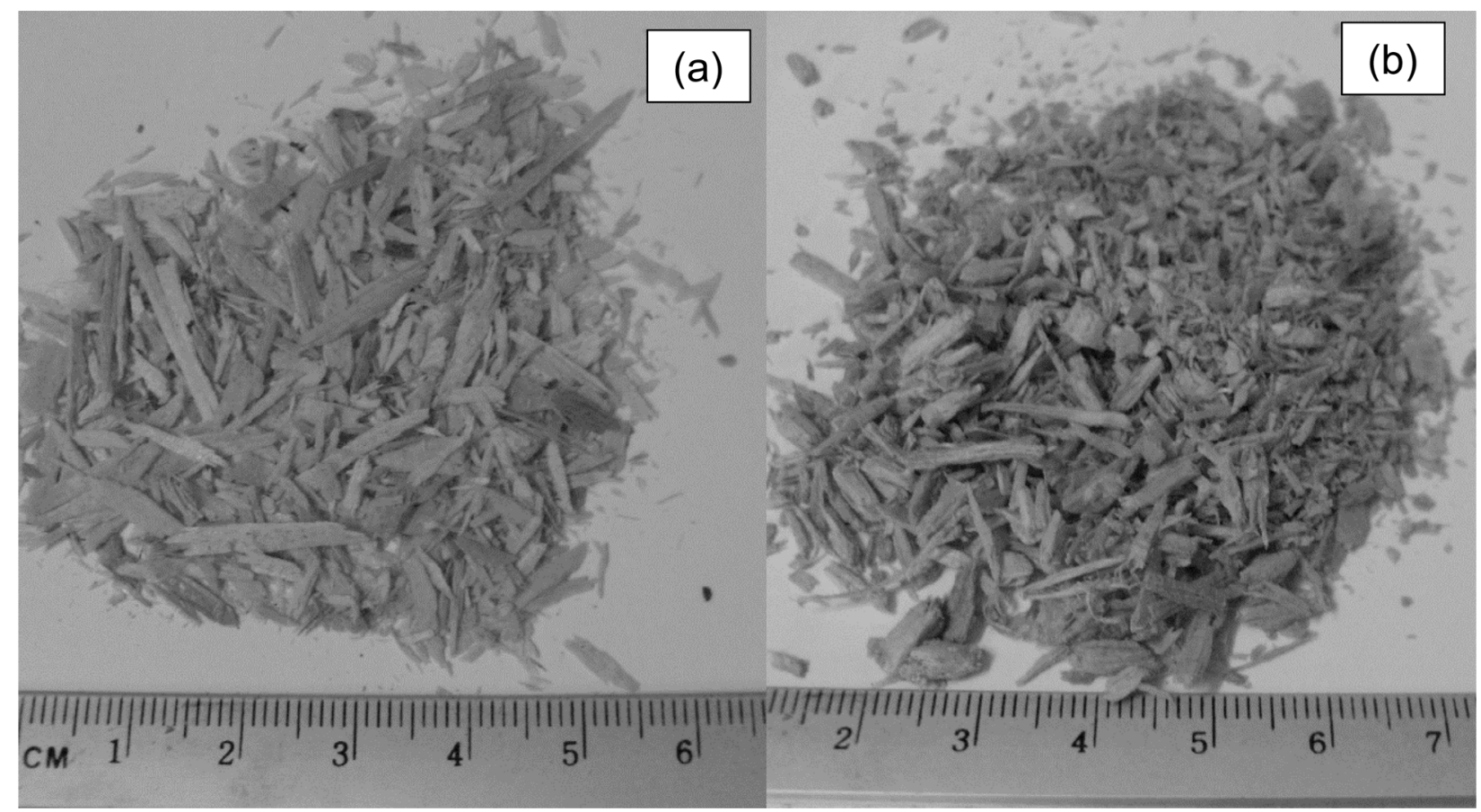

Figura 1. (a) Partículas de Pinus spp. (b) Partículas de Pinus patula.

Figure 1. (a) Particles of Pinus spp. (b) Particles of Pinus patula.

O teor de holocelulose foi obtido por diferença dos demais componentes (extrativos totais, lignina e cinzas).

\section{Delineamento experimental}

Foi utilizado o delineamento inteiramente casualizado (DIC), constituído por 8 tratamentos, compostos por painéis homogêneos com partículas de Pinus patula ou Pinus spp. e 6, 8, 10 e 12\% de adesivo ureia formaldeído. Para cada tratamento foram feitas 3 repetições sendo confeccionados 24 painéis.

Foi realizada uma análise estatística em todos os conjuntos de dados do experimento, em arranjo fatorial $2 \times 4$ com fatores qualitativos (diferentes espécies) e quantitativos (diferentes teores de adesivo).

Posteriormente, foram realizados os testes Shapiro e Wilk (1965); Bartlett (1937), com o intuito de verificar, respectivamente, a normalidade e homogeneidade dos dados. Em decorrência da falta de homogeneidade e normalidade dos dados de Inchamento em espessura, foi necessária a transformação matemática do tipo Box-Cox para a variável.

Em seguida, foi realizada a análise da variância. Nos casos em que não houve interação para os fatores qualitativos foi realizado o teste de médias de Scott e Knott (1974). Já quando constatou-se interação foi realizada a análise de regressão. A análise foi realizada, utilizando o software Sisvar 5.3 Build 77 desenvolvido por Ferreira (2011).

\section{Confecção dos painéis}

Primeiramente, as partículas foram secas em estufa a $60^{\circ} \mathrm{C}$ até atingirem valores de $4 \%( \pm 2 \%)$ de umidade. O grau de umidade das partículas foi determinado por meio de uma termobalança de infravermelho. Com as partículas secas iniciou-se a produção dos painéis, os mesmos com dimensões de $40 \times 40 \times 1,6 \mathrm{~cm}$ (largura, comprimento e espessura). A produção dos painéis foi realizada no Laboratório de Tecnologia da Madeira do Departamento de Engenharia Florestal, na Universidade do Estado de Santa Catarina.

A resina utilizada foi a ureia formaldeído, com teor de sólidos de 69\%. Para a aspersão do adesivo na encoladeira do tipo tambor giratório, foram utilizados copo graduado e uma pistola a ar comprimido. Para a prensagem foram utilizadas duas etapas distintas, na primeira com o objetivo de acomodar as partículas, o material foi submetido a pressão de $5 \mathrm{kgf} / \mathrm{cm}^{2}$ durante 5 minutos, em prensa hidráulica manual. A segunda etapa foi realizada em uma prensa hidráulica automática, onde a prensagem ocorreu sob temperatura de $180^{\circ} \mathrm{C}$, pressão de $40 \mathrm{kgf} / \mathrm{cm}^{2}$, durante 8 minutos. 
Posteriormente a prensagem, os painéis foram climatizados, de acordo com a norma da ABNT (2004), com temperatura de $20 \pm 2^{\circ} \mathrm{C}$ e umidade relativa de $65 \pm 5 \%$.

\section{Testes físicos e mecânicos}

Para os testes físicos de densidade $(5 \times 5 \mathrm{~cm})$, absorção de água em 2 e 24 horas $(15 \times 15 \mathrm{~cm})$, inchamento em espessura em 2 e 24 horas $(15 \times 15 \mathrm{~cm})$, foi utilizada a norma ASTM 1037 (1997). Nos testes mecânicos de ligação interna $(5 \times 5 \mathrm{~cm})$, módulo de elasticidade à flexão estática $(25 \times 5$ $\mathrm{cm})$ e módulo de ruptura à flexão estática $(25 \times 5 \mathrm{~cm})$, foi utilizada a norma DIN (1982).

Por sua vez a razão de compactação foi calculada, por meio da equação:

Em que:

$$
R c=\frac{\text { dpaínel }}{\text { dmadeira }}
$$

$R C$ = Razão de compactação;

dpainel = Densidade aparente do painel;

dmadeira $=$ Densidade básica da madeira.

\section{RESULTADOS E DISCUSSÃO}

\section{Caracterização química}

A baixa quantidade de extrativos totais $(2,84 \%)$ encontrada na madeira de Pinus patula é favorável para a produção de painéis aglomerados, pois, segundo Cloutier (1998), a alta quantidade de extrativos interfere no tempo de cura do adesivo, além de aumentar a propensão dos painéis aglomerados a estouros (Figura 2).

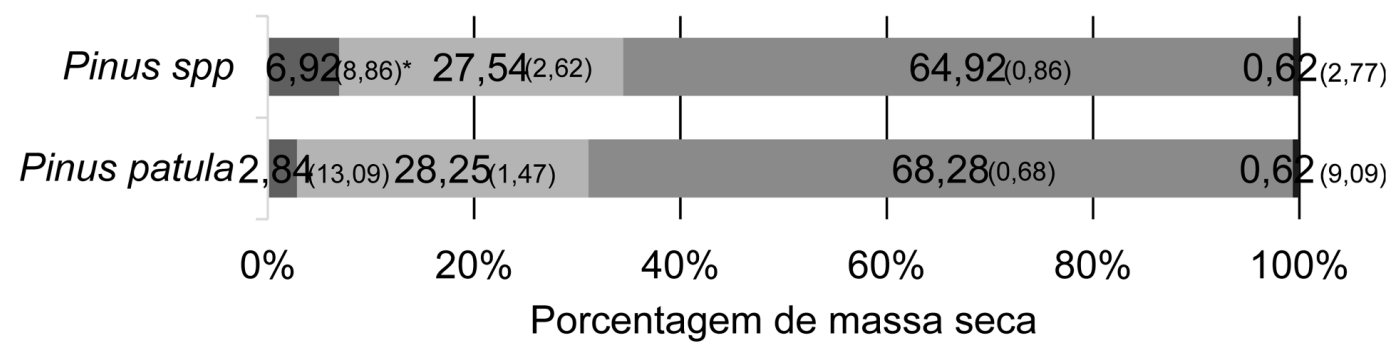

Extrativos Totais $\square$ Lignina $\square$ Holocelulose $\square$ Cinzas

\footnotetext{
* Coeficiente de variação entre parênteses.

Figura 2. Composição química das madeiras de Pinus patula e Pinus spp.

Figure 2. Chemical composition of the wood of Pinus patula and Pinus spp.
}

Os teores de lignina encontrados para o Pinus patula $(28,25 \%)$ e Pinus spp. $(27,54 \%)$ são semelhantes aos encontrados para o mesmo gênero na literatura. Melo et al. (2005) encontraram valores médios de 25,18\% ao estudarem a madeira de Pinus oocarpa. De acordo com Maloney (1993) a lignina pode funcionar como adesivo nos painéis aglomerados, portanto o maior percentual de lignina é desejável para a produção de painéis aglomerados.

Os teores de holocelulose encontrados para a madeira de Pinus patula e Pinus spp. (Industrial) (68,28\% e 64,22\%, respectivamente) assemelham-se aos valores encontrados na literatura para espécies do gênero Pinus. Klock (2000) encontrou 68,00\% para a madeira de Pinus taeda; Rodrigues et al. (2010) encontraram 65,21\% para a madeira de Pinus oocarpa; e Gorski (2014) encontrou 63,09\% para uma mistura de espécies de Pinus.

Para o teor de cinzas as duas espécies apresentaram o mesmo valor $(0,62 \%)$, o qual foi similar aos encontrados por Furtado et al. (2012) que ao estudarem a correlação entre teor de umidade e eficiência energética de resíduos de Pinus taeda em diferentes idades encontraram valores de 0,76\% para teor de cinzas.

Segundo Iwakiri et al. (2005a), o teor de cinzas encontrado está próximo da faixa ideal para a produção de painéis $(0,5 \%)$, essa porcentagem é favorável para a produção de painéis, uma vez que 
os compostos inorgânicos em alta quantidade influenciam no pH dos painéis de madeira aglomerada prejudicando o desenvolvimento de resistência e coesão da linha de cola.

\section{Propriedades físicas}

\section{Densidade aparente}

Para a variável espécie, não houve diferença estatística para os valores de densidade aparente (Tabela 1). Na tabela 1 encontram-se as interações entre as variáveis do estudo. Para a variável espécie, não houve diferença estatística para os valores de densidade aparente.

Tabela 1. Análise das interações entre as variáveis do estudo.

Table 1. Analysis of the interactions between the study variables.

\begin{tabular}{lccc}
\hline \multirow{2}{*}{ Ensaio } & \multicolumn{3}{c}{ p>Fc } \\
\cline { 2 - 4 } & Espécie & Teor de adesivo & Espécie*Teor de adesivo \\
\hline Densidade aparente & 0,9791 & 0,9488 & 0,9966 \\
RC & 0,0000 & 0,0242 & 0,0627 \\
AA 2h & 0,0158 & 0,0000 & 0,7681 \\
AA 24h & 0,0110 & 0,0000 & 0,7354 \\
IE 2h & 0,0006 & 0,0000 & 0,1278 \\
IE 24h & 0,1243 & 0,0000 & 0,2511 \\
Tração perpendicular & 0,0000 & 0,0000 & 0,0000 \\
MOE & 0,0000 & 0,0000 & 0,0693 \\
MOR & 0,0000 & 0,0000 & 0,0528 \\
Parafuso - topo & 0,0825 & 0,0000 & 0,0496 \\
Parafuso - superfície & 0,5393 & 0,0000 & 0,3651 \\
\hline
\end{tabular}

NOTA: Valores de $\mathrm{p}>\mathrm{Fc}$ menores que 0,05 demonstram a ocorrência de diferença significativa/Interação. RC: Razão de Compactação; $A \mathrm{~A} 2 \mathrm{~h}$ : $\mathrm{Ab}$ sorção de água em 2 horas de imersão; AA 24h: Absorção de água em 2 horas de imersão; IE 2h: Inchamento em espessura em 2 horas de imersão; IE 24h: Inchamento em espessura em 24 horas de imersão; MOE: Módulo de elasticidade; MOR: Módulo de ruptura.

Os valores mostraram-se inferiores ao desejado que era de $650 \mathrm{~kg} \cdot \mathrm{m}^{-3}$ (Tabela 2), fato que em partes se explica pelo deslizamento das partículas durante a etapa de prensagem, semelhante ao relatado por Trianoski (2010) e Iwakiri et al., (2005b) em seus estudos.

Tabela 2. Valores médios de densidade aparente e razão de compactação para os diferentes materiais. Table 2. Mean values of bulk density and compression ratio for different materials.

\begin{tabular}{lcccc}
\hline Material & Densidade $\mathbf{( k g \cdot \mathbf { m } ^ { - 3 } \text { ) }}$ & CV (\%) & Razão de compactação (\%) & CV (\%) \\
\hline Pinus patula & $564 \mathrm{a}$ & 4,91 & $1,764 \mathrm{a}$ & 1,90 \\
Pinus spp.(industrial) & $566 \mathrm{a}$ & 7,57 & $1,599 \mathrm{~b}$ & 1,95 \\
\hline
\end{tabular}

NOTA: Médias com letras diferentes em uma mesma coluna diferem estatisticamente entre si pelo Teste de Scott e Knott ao nível de $5 \%$ de probabilidade. CV: Coeficiente de variação.

Para a variável teor de adesivo, os resultados obtidos demonstram que não houve influência do teor de adesivo na densidade aparente dos painéis produzidos (Tabela 3).

Tabela 3. Valores médios de densidade aparente para os diferentes teores de adesivo.

Table 3. Mean values of density for different adhesive contents.

\begin{tabular}{lcccc}
\hline Teor de adesivo (\%) & Densidade $\left(\mathbf{k g} \cdot \mathbf{m}^{-3}\right)$ & CV (\%) & Razão de compactação (\%) & CV (\%) \\
\hline 6 & 547 a & 3,70 & 1,630 a & 3,70 \\
8 & 572 a & 5,57 & 1,707 a & 5,57 \\
10 & $572 \mathrm{a}$ & 2,96 & 1,703 a & 2,96 \\
12 & $568 \mathrm{a}$ & 3,32 & 1,687 a & 3,32 \\
\hline
\end{tabular}

NOTA: Médias com letras diferentes em uma mesma coluna diferem estatisticamente entre si pelo Teste de Scott e Knott ao nível de $5 \%$ de probabilidade. CV: Coeficiente de variação.

Bianche et al. (2012), determinando as propriedades de painéis aglomerados fabricados com partículas de eucalipto (Eucalyptus urophylla), paricá (Schizolobium amazonicum) e vassoura (Sida spp.) também encontraram valores estatisticamente iguais para a densidade aparente dos painéis produzidos com diferentes teores de extrativos. 


\section{Razão de compactação}

Para a variável razão de compactação, as espécies estudadas diferiram estatisticamente entre si (Tabela 2). Os dados obtidos demostraram que o Pinus spp. (Industrial) apresentou valores ideais para essa variável $(1,6)$, que de acordo com Maloney (1993) fica entre 1,3 e 1,6. Em contrapartida os painéis confeccionados com Pinus patula apresentaram valores um pouco superiores ao ideal $(1,76)$. Segundo Maloney (1993) valores de razão de compactação superiores a 1,6 ocasionam uma redução nas propriedades físicas e uma melhora nas propriedades mecânicas do painel. Fato que pode ser explicado pela geometria diferenciada das partículas entre as espécies, as mesmas oriundas da indústria, apresentavam-se com aspecto mais fino e alongado, o que provavelmente permitiu uma melhor acomodação das partículas.

Por sua vez a váriavel teor de adesivo não apresentou diferença estatística (Tabela 3), fato que indica que a quantidade de adesivo não interfere nessa variável.

Esse comportamento também foi observado por Trianoski et al. (2014) ao avaliarem painéis aglomerados de Toona ciliata produzidos com diferentes densidades e teores de resina.

\section{Absorção de água em 2 e 24 horas}

Os valores de absorção de água, em 2 e 24 horas apresentaram diferença estatística entre as diferentes espécies e teores de resina, contudo não ocorreu interação significativa entre as variáveis. Desse modo, os resultados serão apresentados separadamente para as diferentes variáveis. Para a variável espécie, o Pinus patula apresentou menores percentuais de absorção de água, em comparação ao Pinus spp. (industrial) (Tabela 4).

Tabela 4. Valores de absorção de água 2 e 24 horas e inchamento em espessura (IE) após 2 e 24 horas de imersão em água para os diferentes materiais.

Table 4. Values of water absorption of 2 to 24 hours and thickness swelling (IE) after 2 and 24 hours immersion in water for different materials.

\begin{tabular}{lcccc}
\hline Material & AA 2h (\%) & CV (\%) & AA 24 h (\%) & CV (\%) \\
\hline Pinus patula & 105,79 a & 15,78 & 118,50 a & 15,56 \\
Pinus spp.(industrial) & 113,31 b & 12,94 & 127,06 b & 11,96 \\
\hline Material & IE 2h (\%) & CV (\%) & IE 24 h (\%) & CV (\%) \\
\hline Pinus patula & 20,75 a & 3,16 & 26,75 a & 6,85 \\
Pinus spp.(industrial) & 20,64 b & 3,46 & 28,84 a & 6,28 \\
\hline
\end{tabular}

NOTA: Médias com letras diferentes em uma mesma coluna diferem estatisticamente entre si pelo Teste de Scott e Knott ao nível de $95 \%$ de probabilidade. AA: Absorção de água; IE: Inchamento em Espessura; CV: Coeficiente de variação.

Os altos valores de absorção de água encontrados para os dois materiais, em parte, são explicados pela baixa densidade da madeira das espécies utilizadas, fato que, segundo Iwakiri et al. (2005a), ocasiona uma maior liberação das tensões de compressão, imposta pela a elevada razão de compactação ocasionada durante a prensagem, o que por sua vez, proporciona maiores taxas de absorção de água. Porém Brito (1995) ressalta que os valores de absorção de água podem ser influenciados por uma série de fatores, tais como: densidade dos painéis, eficiência na aplicação da cola e condições de prensagem.

Um fator que contribuiu significativamente, para os elevados valores encontrados foi a não adição de parafina, uma vez que sua adição em painéis de madeira aglomerada reduz, drasticamente, a higroscospicidade do material. Guimarães Jr. et al. (2013) constatou em seu estudo que a adição de $1 \%$ de parafina promove uma redução de, aproximadamente, $47 \%$ na absorção de água em 2 horas.

Outro fator, que pode ter contribuído significativamente para os elevados percentuais de absorção de água encontrados no estudo foi a característica das partículas que apresentavam menor comprmento e maior espessura, o que resulta em uma maior proporção de partículas posicionadas verticalmente durante a formação do painel De acordo Moslemi (1974), isso contribui para maior instabilidade dimensional dos painéis.

Por sua vez, quando a variável analisada é a quantidade de adesivo, os dados demostraram que, com o aumento do teor de adesivo ocorreu uma redução dos valores médios de absorção de água (Figura 3a). Uma vez que as maiores médias de absorção de água foram encontradas nos tratamentos com $6 \%$ de adesivo ureia formaldeído e, os menores valores, nos tratamentos com $12 \%$ do mesmo adesivo. 

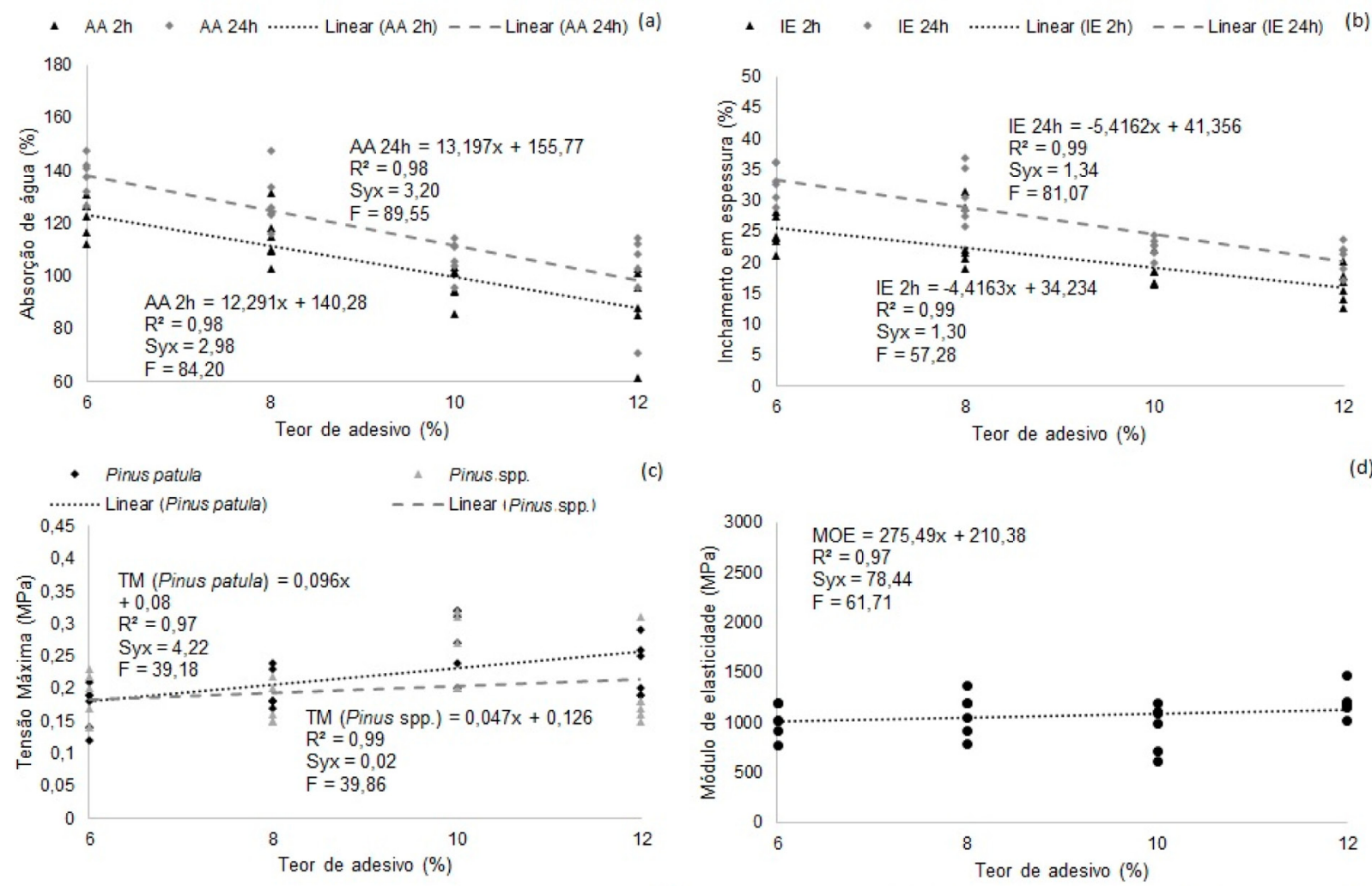

(c)
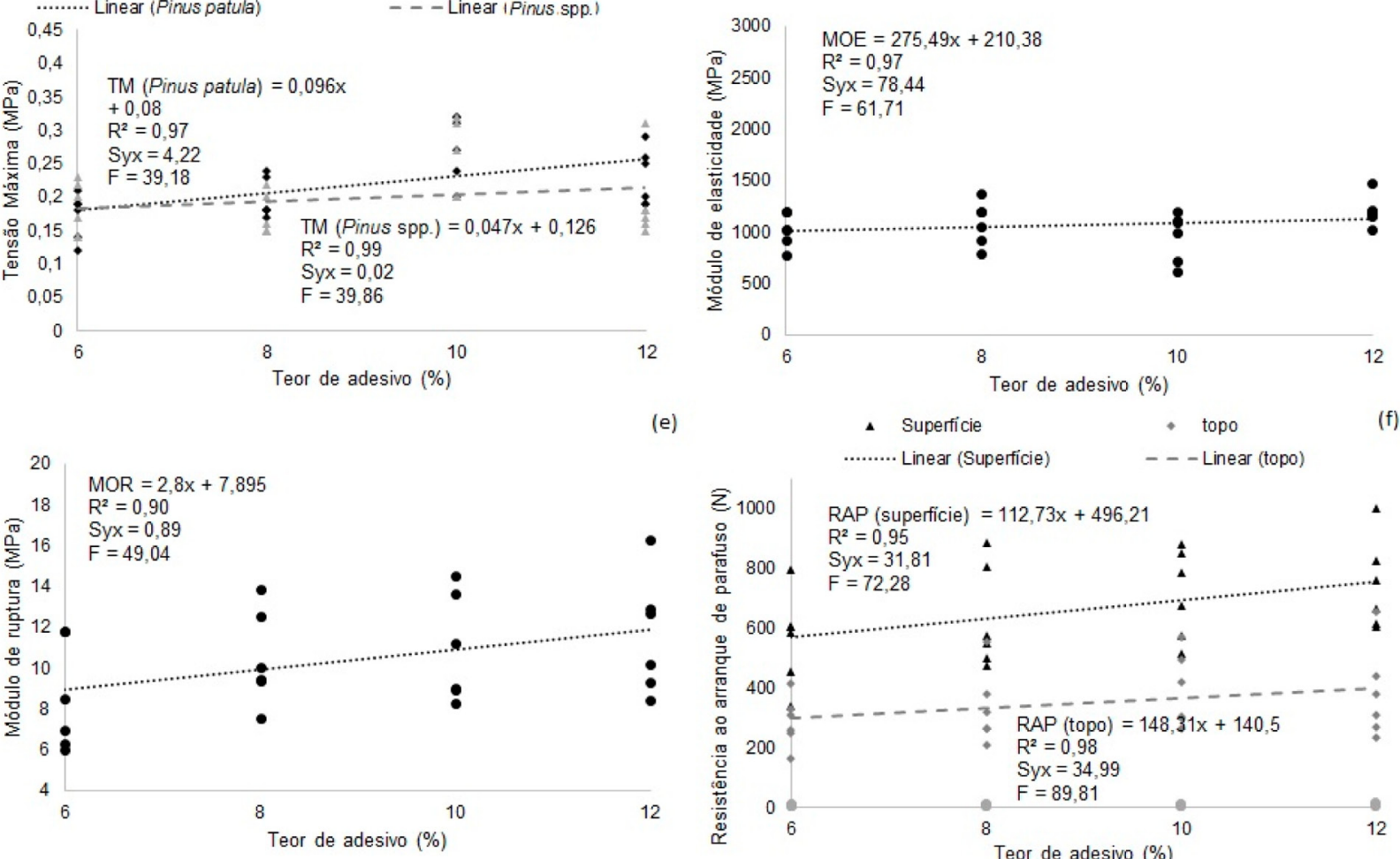

(e)

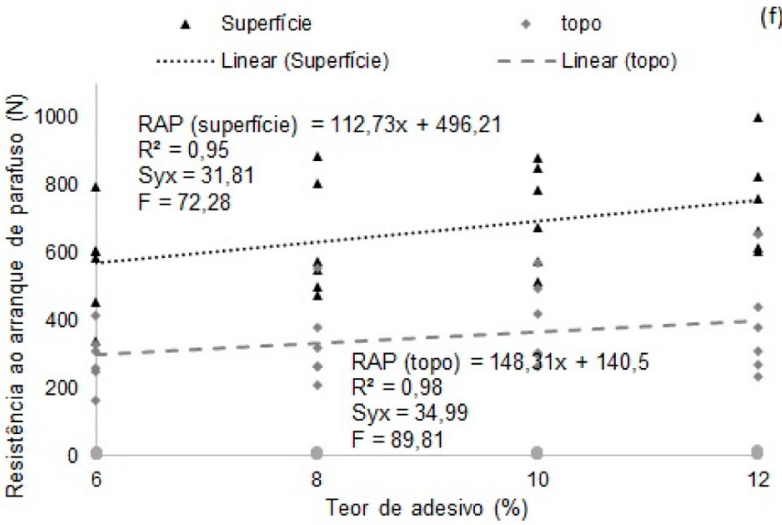

Figura 3. (a) Regressão linear para o ensaio de absorção de água (b) Regressão linear para o ensaio de inchamento em espessura(c) Análise de regressão para a variável tração perpendicular à superfície. (d) Análise regressão para a ensaio de módulo de elasticidade (e) Análise regressão para a ensaio de módulo de ruptura. (f) Análise de regressão para o ensaio de Resistência ao arranque de parafuso *Para as análises de regressão considerou-se os teores de adesivo.

Figure 3. (a) Linear regression to the water absorption test. (b) Linear regression for thickness swelling test. (c) Regression analysis for the variable traction perpendicular to the surface. (d) Regression analysis for the elasticity modulus. (e) Regression analysis for the modulus of rupture test. (f) Regression analysis for the screw starting Resistance test. * For the regression analyses the adhesive contents were considered.

Segundo Albuquerque (2002), isso é explicado pelo fato de que, o aumento no teor de adesivo ocasiona uma consolidação mais efetiva das partículas, uma vez que há um volume superior de adesivo por partícula, condição que além de reduzir os espaços vazios, dificulta o contato da água com o material lenhoso.

Para o teste de inchamento em espessura, em 2 horas as análises estatísticas não indicaram interação entre os fatores, contudo para a variável espécie ocorreu diferença estatística, onde as médias dos painéis produzidos com Pinus patula apresentaram-se estatisticamente superiores aos dos painéis produzidos com Pinus spp. (industrial). Para o ensaio de inchamento em espessura em 24 horas a análise estatística não indicou diferença significativa (Tabela 4).

Para os diferentes teores de ureia formaldeído, a análise de regressão demonstra que o ponto de máxima eficiência técnica para os dois ensaios se encontra com a aplicação de $12 \%$ de adesivo e que os valores menos satisfatórios são encontrados com a aplicação de $6 \%$ de adesivo (Figura 3b), 
indicando que o aumento nos teores de ureia formaldeído nos painéis ocasiona uma redução no inchamento em espessura dos mesmos.

De acordo com a norma CS (1968), com exceção dos painéis produzidos com Pinus spp. (Industrial) e com $6 \%$ de ureia formaldeído, os valores encontrados no estudo atenderam aos requisitos da norma que determina valor máximo de 35\% para inchamento em espessura em 24 horas. Cabe salientar que os valores poderiam ser ainda menores caso fosse realizada a adição de parafina. Segundo Guimarães Jr. et al. (2013) a adição de 1\% de parafina na produção de painéis de madeira aglomerada ocasiona uma redução de aproximadamente 10,9\% no inchamento em espessura.

\section{Propriedades mecânicas}

\section{Tração perpendicular}

Os resultados de tração perpendicular demonstraram que ocorreu interação entre as variáveis espécie e teor de adesivo, com a análise dos resultados foi possível observar que com o aumento nos teores de adesivo ocorreu um incremento na resistência média dos painéis, fato semelhante ao encontrado por Santos et al. (2009); Iwakiri et al. (2000b); Mendes et al. (2012), em estudos com painéis de madeira aglomerada confeccionados utilizando diferentes teores de ureia formaldeído. Segundo Santos et al. (2009), esse incremento pode ser atribuído à maior disponibilidade de adesivo por unidade de área das partículas.

Como é possível observar na Figura 3c, em todos os teores de adesivo os painéis confeccionados utilizando partículas de Pinus patula apresentaram maiores valores que os confeccionados com partículas de Pinus spp. para essa propriedade.

Em relação a norma CS (1968), apenas os painéis produzidos com Pinus patula e 12\% de adesivo apresentaram valores superiores a 0,42 MPa que corresponde ao valor mínimo exigido pela norma. Contudo, os resultados foram semelhantes e muitas vezes superiores aos encontrados na literatura.

Guimarães Jr. et al. (2013) encontraram o valor de 0,27 MPa para painéis confeccionados com Pinus oocarpa utilizando $8 \%$ de ureia-formaldeído e densidade nominal de $700 \mathrm{~kg} . \mathrm{m}^{-3}$; Pedrazzi et al. (2006) encontraram valores de 0,17 e 0,13 Mpa para painéis de Eucalyptus saligna com a utilização de $8 \%$ de ureia-formaldeído e densidade nominal de $650 \mathrm{~kg} \cdot \mathrm{m}^{-3}$ e Mendes et al. (2010) que encontraram valores de 0,19 MPa para painéis produzidos utilizando bagaço de cana com $8 \%$ de ureia-formaldeído e com densidade de $700 \mathrm{~kg} \cdot \mathrm{m}^{-3}$.

\section{Flexão estática}

Para o teste de flexão estática os resultados demonstraram que não ocorreu interação entre as variáveis espécie e teor de adesivo. Para a variável espécie, os painéis produzidos utilizando partículas de Pinus spp. (Industrial), apresentaram-se estatisticamente superiores tanto para MOE quanto para MOR (Tabela 5), o que pode ser atribuído a característica das partículas, uma vez que as partículas de Pinus spp. (Industrial) eram mais compridas e menos espessas que as de Pinus patula. Geimer e Price (1978) relatam que as propriedades de flexão estática dos painéis de madeira aglomerada aumentam diretamente com o comprimento das partículas, em decorrência das características geométricas das próprias partículas e sua capacidade em suportar esforços de flexão.

Tabela 5. Valores de flexão estática e arranchamento de parafuso para as diferentes espécies.

Table 5. Bending values and screw pullout for different species.

\begin{tabular}{lcccc}
\hline Material & MOE (MPa) & CV (\%) & MOR (MPa) & CV (\%) \\
\hline Pinus patula & 1345,87 a & 27,9 & 12,86 a & 32,1 \\
Pinus spp. (industrial) & $1855,28 \mathrm{~b}$ & 25,3 & $16,90 \mathrm{~b}$ & 34,66 \\
\hline Material & AP Superfície (N) & CV (\%) & AP Topo (N) & CV (\%) \\
\hline Pinus patula & $778,31 \mathrm{a}$ & 50,49 & $540,68 \mathrm{a}$ & 28,5 \\
Pinus spp. (industrial) & $758,39 \mathrm{a}$ & 39,48 & $481,69 \mathrm{a}$ & 25,88 \\
\hline
\end{tabular}

Nota: Médias com letras diferentes em uma mesma coluna diferem estatisticamente entre si pelo Teste de Scott e Knott ao nível de $95 \%$ de probabilidade; MOE: Módulo de Elasticidade; CV: Coeficiente de variação; MOR: Módulo de Ruptura; AP: Arrancamento de Parafuso.

Para a variável teor de adesivo foi possível observar que o aumento nos seus teores ocasionou uma melhora nas propriedades de MOE e MOR, sendo que a máxima eficiência técnica foi encontrada 
com a utilização de 12\% de ureia formaldeído (Figuras 3d e 3e). Segundo Mendes et al. (2010), isso pode ser explicado pelo fato de que quanto maior for a quantidade de adesivo, aplicada na produção dos painéis, melhor será a ligação entre as partículas, o que resulta em maior resistência à flexão.

Em relação a norma CS (1968) nenhum dos tratamentos atingiu os valores mínimos estipulados para o MOE $(2450 \mathrm{~N})$, em contrapartida para o MOR as médias das duas espécies apresentaram-se nos padrões exigidos pela mesma $(11,2 \mathrm{~N})$ e, entre os diferentes teores de adesivo somente as médias dos painéis com $6 \%$ de adesivo não atenderam aos requisitos da norma. Os resultados encontrados no estudo também foram semelhantes aos encontrados na literatura como no estudo de Mendes et al. (2010) que encontraram médias entre 913,48 N e 1062,59N para MOE e entre 11,37 e 14,38 para MOR em estudo de painéis produzidos com diferentes teores de ureia formaldeído, com madeira de Eucalipto e bagaço de cana.

\section{Resistência ao arrancamento de parafuso}

Os resultados do ensaio de resistência ao arranque de parafuso indiciaram que não ocorreu interação entre as variáveis espécie e teor de adesivo. Para a variável teor de adesivo o aumento nos teores de ureia formaldeído ocasionou uma melhora nas propriedades dos painéis, sendo que o ponto de máxima eficiência técnica foi encontrado com utilização de 12\% de ureia-formaldeído, tanto para o ensaio de topo, quanto para o de superfície (Figura 3f). Em contrapartida, para a variável espécie, os resultados não aferiram diferença estatística (Tabela 5).

Em relação a norma ABNT (2006) nenhum dos tratamentos atingiu os valores recomendados pela norma que estabelece limites mínimos de $1020 \mathrm{~N}$ para arrancamento de superfície e de $800 \mathrm{~N}$ para topo. Contudo, os resultados foram semelhantes aos encontrados em outros estudos como o de Melo e Del Menezzi (2010) que encontraram valores no intervalo de 710 a 966 N para painéis de Eucalyptus grandis produzidos com $8 \%$ de ureia formaldeído.

Os baixos valores encontrados para essa propriedade podem ser atribuídos a baixa densidade dos painéis. Segundo Cunha et al. (2014), painéis com alta densidade apresentam melhores valores de resistência para o ensaio de arrancamento de parafuso que painéis de baixa densidade.

Portanto, em estudos futuros, para que se atinja os valores mínimos exigidos pelas normas, novas composições de painéis devem testadas. Pode-se destacar, o aumento da proporção de resina, o aumentar a densidade nominal do painel ou a incorporação de painéis heterogêneos compostos por mix de partículas de Pinus patula e Pinus spp.

\section{CONCLUSÕES}

- A densidade nominal ( $650 \mathrm{~kg} \cdot \mathrm{m}^{-3}$ ) dos painéis produzidos não foi atingida. O valor médio obtido foi de $565 \mathrm{~kg} \cdot \mathrm{m}^{-3}$.

- A razão de compactação obtida para Pinus spp. estava na faixa considerada ideal (entre 1,3 e 1,6), por sua vez a obtida para a madeira de Pinus patula foi levemente superior a recomendada.

- Todos os tratamentos, com exceção dos painéis produzidos com Pinus spp. (Industrial) e com 6\% de ureia formaldeído atingiram os valores exigidos pela norma CS (1968) para inchamento em espessura em 24 horas.

- Para tração perpendicular, somente o tratamento produzido com Pinus patula e com $12 \%$ de adesivo atendeu ao mínimo exigido pela norma CS (1968)

- Em relação ao MOE nenhum dos tratamentos atingiu o mínimo exigido pela norma CS (1968). Já em relação ao MOR somente os tratamentos confeccionados com $6 \%$ de adesivo não atingiram o valor mínimo exigido pela norma.

- Para arranamento de parafuso, nenhum dos tratamentos atingiu o mínimo exigido pela norma ABNT (2006).

- A madeira de Pinus patula apresenta potencial para produção de painéis de madeira aglomerada, tendo em vista que os valores obtidos para a espécie no estudo foram em grande parte superiores aos de Pinus spp. (Industrial) e semelhantes aos encontrados na literatura.

- Com exceção das propriedades de MOR e MOE, nas demais propriedades físicas e mecânicas analisadas os painéis produzidos com Pinus patula apresentaram valores estatisticamente iguais ou melhores aos produzidos com painéis de Pinus spp. (Industrial). 
Rios et al. - Avaliação do potencial da madeira de Pinus patula

Schltdl. \& Cham para a produção de painéis de madeira aglomerada

- Dos painéis produzidos com madeira de Pinus patula, pode-se destacar o tratamento com 12\% de adesivo, pois o aumento nos teores de adesivo ocasionou um incremento na qualidade dos painéis, influenciando diretamente a grande maioria das propriedades estudadas.

\section{AGRADECIMENTOS}

As empresas Seiva S.A. Florestas e Indústrias e Bonet Madeiras e Papéis Ltda pelo fornecimento da matéria prima utilizada para a produção dos painéis aglomerados. À FAPESC, pelo apoio financeiro e institucional.

Ao pesquisador Davi Junges (in memoriam) pela colaboração para a realização deste trabalho.

\section{REFERÊNCIAS BIBLIOGRÁFICAS}

ABNT. ASSOCIAÇÃO BRASILEIRA DE NORMAS TÉCNICAS. NBR 14660: Madeira - Amostragem e preparação para análise. Rio de Janeiro; 2004. 7p.

ABNT. ASSOCIAÇÃO BRASILEIRA DE NORMAS TÉCNICAS. NBR 14810: Chapas de madeira aglomerada. 2006. $51 \mathrm{p}$.

ALBUQUERQUE, C. E. C. Interações de variáveis no ciclo de prensagem de aglomerados. 2002. 150 p. Tese (Doutorado em Ciências Florestais) Universidade Federal do Parará, Curitiba, 2002.

ABRAF. ASSOCIAÇÃO BRASILEIRA DE PRODUTORES DE FLORESTAS PLANTADAS, Anuário Estatístico da ABRAF 2010: Ano base 2009. Brasília: 2010. 140 p.

ASTM. AMERICAN SOCIETY FOR TESTING AND MATERIALS. Annual book of ASTM: D-1037 - Standard methods of evaluating properties of wood-base fiber and particles materials. Philladelphia, 2002

BARTLETT, M. S. Properties of sufficiency and statistical tests. Proceedings of the Royal Society of London. Series A, Mathematical and Physical Sciences, v. 160, n. 901, p. 268-282, 1937.

BIANCHE, J. J.; CARNEIRO, A. C. O.; VITAL, B. R.; PEREIRA, F. A.; SANTOS, R. C.; SORATTO, D. N. Propriedades de painéis aglomerados fabricados com partículas de eucalipto (Eucalyptus urophylla), paricá (Schizolobium amazonicum) e vassoura (Sida spp.). Cerne, Lavras, v. 18, n. 4, p. 623-630, 2012.

BRITO, E. O. Produção de chapas de partículas de madeira a partir de maravalhas de Pinus elliottii Engelm. Var. elliottii plantado no sul do Brasil. Curitiba: 1995. 123 p. Tese (Doutorado em Ciências Florestais) Setor de Ciências Agrárias, Universidade Federal do Paraná, Curitiba, 1995.

CARVALHO, A. G.; ZANUNCIO, A. J. V.; MENDES, R. F; MORI, F. A.; SILVA, M. G.; MENDES, L. M. Adesivos tânicos de Stryphnodendron adstringens (Mart.) Coville na produção de painéis aglomerados. Revista Árvore, Viçosa, v. 38, n. 1, p. 195-202, 2014.

CLOUTIER, A. Oriented strandboard (OSB): raw material, manufacturing process, properties of wood-base fiber and particle materials. In: INTERNATIONAL SEMINAR ON SOLID WOOD PRODUCTS OF HIGH TECHNOLOGY. 1998, Belo Horizonte. Anais... Viçosa: SIF, 1998, p. 173-185.

CS. COMMERCIAL STANDARD. CS 236-66: mat formed wood particleboard. [S.I.], 1968.

CUNHA, A. B.; LONGO, B. L.; RODRIGUES, A. A.; BREHMER, D. R. Produção de painéis de madeira aglomerada de Eucalyptus benthamii, Eucalyptus dunnii e Eucalyptus grandis. Scientia Forestalis, Piracicaba, v. 42, n. 102, p. 259-267, 2014. 
DAFF. DEPARTMENT OF AGRICULTURE, FORESTRY AND FISHERIES, Report on commercial timber resources and primary roundwood processing in South Africa 2008/9. Pretoria, 2010.

DIN - NORMEN FÜR HOLZFASERPLATEN SPANPLATTEN SPERRHOLZ. DIN 52362: Testing of wood chipboards bending test, determination of bending strength. Berlin, 1982. p. 39-40.

FURTADO, T. S.; FERREIRA, J. C.; BRAND, M. A.; NEVES, M. D. Correlação entre teor de umidade e eficiência energética de resíduos de Pinus taeda em diferentes idades. Revista Árvore, Viçosa, v. 36, n. 3, p. 577-582, 2012.

GEIMER, R. L.; PRICE, E. W. Construction variables considered in the fabrication of structural flakeboard. Washington: USDA Forest Service, 1978. p. 69-80. (WO General Technical Report, 5).

GORSKI, L. Painéis de partículas orientadas (OSB) da madeira de Pinus spp. E Eucalyptus Benthamii. 2014. 146 p. Dissertação (Mestrado em Engenharia Florestal) - Universidade do Estado de Santa Catarina, Lages, 2014.

GUIMARÃES JR., J. B.; ARAÚJO, B. L. M.; LOPES, O. P.; MENDES, R. F.; MENDES, L. M. Produção de painéis aglomerados de madeira de desrama de Acacia mangium. Pesquisa florestal brasileira, Colombo, v. 33, n. 76, p. 387-391, 2013.

KLOCK, U. Qualidade da madeira juvenil de Pinus maximinoi H. E. Moore. 2000. 324 p. Tese (Doutorado em Ciências Florestais) - Universidade Federal do Paraná, Curitiba, 2000.

IWAKIRI, S.; CAPRARA, A. C.; SAKS, D. C. O.; GUISANTES, F. P.; FRONZINI, J. A.; KRAMBECK, L. B. P.; RIGATTO, P. A. Produção de painéis aglomerados de alta densificação com uso de resina melamina-uréiaformaldeído. Cerne, Lavras, v. 11, n. 4, p. 323-328, 2005b.

IWAKIRI, S.; CUNHA, A. B.; ALBUQUERQUE, C. E. C.; GORNIAK, E.; MENDES, L. M. Resíduos de serraria na produção de painéis de madeira aglomerada de Eucalipto. Scientia Agrária, Curitiba, v. 1, n. 1-2, p. 23-28, 2000b.

IWAKIRI, S.; SILVA, J. C.; SILVA, J. R. M.; ALVES, C. R.; PUEHRINGER, C. A. Produção de compensados de Pinus taeda L. e Pinus oocarpa Schiede com diferentes formulações de Uréia-Formaldeído. Revista Árvore, v. 26, n. 3, p. 371-375, 2002.

IWAKIRI, S. et al. Painéis de Madeira. Curitiba: FUPEF, p. 196. $2005 a$.

MALONEY, T. M. Modern particleboard and dry-process fiberboard manufacturing. 2.ed. São Francisco: Miller Freeman, 1993. 689 p.

MELO, R. R.; DEL MENEZZI, C. H. S. Influência da massa específica nas propriedades físico-mecânicas de painéis aglomerados. Silva Lusitana, Lisboa, v. 18, n. 1, p. 59-73, 2010.

MELO, S. A. L.; NASCIMENTO, E. A.; MELO, D. C. Análise da madeira de Pinus oocarpa parte I: estudo dos constituintes macromoleculares e extrativos voláteis. Revista Árvore, Viçosa, v. 29, n. 3, p. 461-470, 2005.

MENDES, R. F. et al. Associação de eucalipto e pinus na produção de painéis aglomerados de bagaço de cana. In: ENCONTRO BRASILEIRO EM MADEIRAS E EM ESTRUTURAS DE MADEIRA, 12, 2010, Lavras. Anais... Lavras, 2010.

MENDES, R. F.; GUIMARÃES JR., J. B.; SANTOS, R. C.; CÉSAR, A. A. S. Efeito da associação do bagaço de cana, do tipo e do teor de adesivo na produção de painéis aglomerados. Ciência Florestal, Santa Maria, v. 22, n. 1, p. $161-170,2012$. 
Rios et al. - Avaliação do potencial da madeira de Pinus patula

Schltdl. \& Cham para a produção de painéis de madeira aglomerada

MOSLEMI, A. A. Particleboard. Illionis: Southern Illionis University, 1974. v. 2, 245 p.

PEDRAZZI, C.; HASELEIN, C. R.; SANTINI, E. J.; SCHNEIDER, P. R. Qualidade de chapas de partículas de madeira aglomerada fabricadas com resíduos de uma indústria de celulose. Ciência Florestal, Santa Maria, v. 16, n. 2, p. 201-212, 2006.

RODRIGUES, C. K.; HILLIG, E.; MACHADO, G. O. Análise química da madeira de Pinus oocarpa. In: ANAIS DA SEMANA DE INTEGRAÇÃO ENSINO, PESQUISA E EXTENSÃO. 2009. Guarapuava. Anais... Guarapuava, 2010 .

SANTOS, R. C.; MENDES, L. M.; MORI, F. A.; MENDES, R. F. Chapas de partículas aglomeradas a partir de resíduos gerados após a extração de óleo da madeira de candeia (Eremanthus erytropapus). Scientia Forestalis, Piracicaba, v. 37, n. 84, p. 437-446, 2009.

SCOTT, A. J., KNOTT, M. A cluster analysis method for grouping means in the analysis of variance. Biometrics, Raleigh, v. 30, n. 3, p. 507-12, 1974.

SHAPIRO, S. S.; WILK, M. B. An analysis of variance test for normality (complete samples). Biometrika, Cambridge, p. 591-611, 1965.

SHIMIZU, J. Y. Pinus patula. Embrapa Florestas: Sistemas de Produção, 5. Versão Eletrônica. Nov. 2005.

TAPPI. TECHNICAL ASSOCIATION OF THE PULP AND PAPER INDUSTRY. TAPPI T222: Acid-insoluble lignin in wood and pulp, Test Method. Atlanta, 2002. 5 p.

TAPPI. TECHNICAL ASSOCIATION OF THE PULP AND PAPER INDUSTRY. TAPPI 413: Ash in wood, pulp, paper and paperboard: combustion at $900^{\circ} \mathrm{C}$, Test Method. Atlanta, 1993. 4 p.

TRIANOSKI, R. Avaliação do potencial de espécies florestais alternativas de rápido crescimento para produção de painéis de madeira aglomerada. Curitiba, 2010. 260 p. Dissertação (Mestrado em Engenharia Florestal) - Setor de Ciências Agrárias, Universidade Federal do Paraná. 2010.

TRIANOSKI, R.; IWAKIRI, S.; MATOS, J. L. Avaliação de painéis aglomerados de Toona ciliata produzidos com diferentes densidades e teores de resina. Madera y Bosques, México, v. 20, n. 3, p. 49-58, 2014.

Recebido em 04/03/2015

Aceito para publicação em 17/12/2015 